Supporting information for the manuscript entitled

\title{
Characterization of Modified Phenol Formaldehyde Resole Resins Synthesized in-situ with Various Boron Compounds
}

\author{
Yubo Chai ${ }^{\text {a, }}$ Junliang Liu ${ }^{a}$, Yong Zhao ${ }^{b}$, Ning Yan ${ }^{\text {* }}$ \\ a. Research Institute of Wood Industry, Chinese Academy of Forestry, Beijing, China 100091 \\ b. Faculty of Forestry, University of Toronto, 33 Willcocks Street, Toronto, ON. Canada M5S 3B3 \\ * Corresponding author: Tel:+1-416-946-8070, +1-416-978-3834, Email address: ning.yan@utoronto.ca
}


Table S1. Assignments of chemical shifts for the liquid-state ${ }^{13} \mathrm{C}$ NMR spectra of lab made control PF and boron compounds modified PF resins ${ }^{31,37-40}$

\begin{tabular}{|c|c|}
\hline Chemical shifts (ppm) & Assignment \\
\hline $166-168$ & $\mathrm{C}=\mathrm{O}$ of carbonyl \\
\hline $153.5-157.2$ & phenoxy, alkylated in ortho position \\
\hline $158.0-159.5$ & phenoxy, alkylated in para position \\
\hline $129.0-130.4$ & phenolic substituted para aromatic carbons \\
\hline $126.0-128.1$ & phenolic substituted ortho aromatic carbons \\
\hline $120.0-124.0$ & phenolic unsubstituted para aromatic carbons \\
\hline $114.0-119.0$ & phenolic unsubstituted ortho aromatic carbons \\
\hline 86-93.7 & formaldehyde oligomers \\
\hline $69.0-73.0$ & methylene ether bridges between phenol, $\mathrm{Ph}-\mathrm{CH}_{2}-\mathrm{O}-\mathrm{CH}_{2}-\mathrm{Ph}$ \\
\hline 63.1-64.7 & para methylol, $p-\mathrm{Ph}-\mathrm{CH}_{2} \mathrm{OH}$ \\
\hline $60.4-62.8$ & ortho methylol, $o-\mathrm{Ph}-\mathrm{CH}_{2} \mathrm{OH}$ \\
\hline $48.7-50$ & Methanol, $\mathrm{CH}_{3} \mathrm{OH}$ \\
\hline $39.1-41$ & para-para methylene link, $p, p-\mathrm{Ph}-\mathrm{CH}_{2}-\mathrm{Ph}$ \\
\hline 34.6-35.0 & ortho-para methylene link, $o, p-\mathrm{Ph}-\mathrm{CH}_{2}-\mathrm{Ph}$ \\
\hline 29.3-29.6 & ortho-ortho methylene link, o,o- $\mathrm{Ph}-\mathrm{CH}_{2}-\mathrm{Ph}$ \\
\hline
\end{tabular}

